\title{
Estudos para incentivar soluções sociocriativas por meio de tecnologias sociais na America Latina ${ }^{1}$
}

\author{
Estudios para incentivar soluciones socio creativas a través de tecnologías \\ sociales en América Latina
}

\author{
Studies to encourage socio-creative solutions through social technologies \\ in Latin America
}

\author{
Rita de Castro Engler ${ }^{2}$ \\ Nadja Maria Mourão3
}

\begin{abstract}
Resumo
No mundo atual, as tecnologias sociais se tornaram alternativas para solução de problemas estruturais da sociedade, de forma simples, de baixo custo, replicáveis e apoiadas por instituições. Elas estão fundamentas em duas bases para sua propagação: a participação de pessoas das comunidades que as desenvolvem, e a sustentabilidade nas soluções apresentadas. No Brasil e países vizinhos, são encontrados exemplos de tecnologias sociais, certificadas pela Fundação Banco do Brasil, que geram soluções sociocriativas. Pelo perfil associativo e solidário, essas tecnologias sociais são mecanismos que promovem a autogestão, fator relevante, em tempos de crise econômica. O objetivo dessa pesquisa é analisar o desenvolvimento dos projetos de tecnologias sociais, de forma que possam ser replicados em outras comunidades da América Latina, em soluções sociocriativas. A metodologia compreende pesquisa bibliográfica de contextos temáticos. As questões das comunidades de fronteiras podem ser compreendidas pela semelhança das dificuldades territoriais, que são abrandadas por fatores culturais. É analisado o potencial de compartilhamento das tecnologias sociais em inteligência sociocriativa. Esta pesquisa é um estudo das possibilidades de gerar soluções em conjunto, em um mundo onde, as opiniões diferentes de questões políticas e culturais, geram conflitos e exclusão. A inteligência sociocriativa propõe a conexão da diversidade de olhares e pensamentos, inclusive opostos, para criar realidades mais inclusivas e sustentáveis. Modelos de tecnologias sociais podem promover o diálogo entre comunidades, inclusive entre fronteiras, em experiências semelhantes. Um dos exemplos é o projeto "10caminhos" que propõe solução em geração de renda para as populações em situação de vulnerabilidade econômica ao crime. Tem como objetivo organizar o primeiro distrito criativo na tríplice fronteira Brasil, Argentina e Paraguai. Assim, em Foz do Iguaçu/Brasil, foi criado o primeiro núcleo na comunidade da Vila $\mathrm{C}$ - uma tecnologia social em modelo sociocriativo. Espera-se que este se torne um distrito criativo, um referencial da tríplice fronteira Latina Americana.
\end{abstract}

Palavras-chave: Tecnologias sociais, soluções sociocriativas, fronteira, geração de renda, inclusão social.

\footnotetext{
${ }^{1}$ Artigo apresentado no Simpósio Temático - Diálogos interculturais de fronteiras: perspectivas teóricas e experiências compartilhadas, durante o II Seminário Latino-Americano de Estudos em Cultura - SEMLACult em Foz do Iguaçu/PR, Brasil, 2018.

${ }^{2}$ PhD Gestão de Inovação Tecnológica/ECP França; Professora PPGD-UEMG; Coordenadora do CEDTec da UEMG; Cidade de Belo Horizonte, Estado de Minas Gerais, Brasil; rita.engler@gmail.com

3 Mestra e Doutoranda em Design; Centro de Estudos em Design e Tecnologia da Escola de Design- CEDTec, Universidade do Estado de Minas Gerais-UEMG; Cidade de Belo Horizonte, Estado de Minas Gerais, Brasil; nadjamourao@gmail.com
} 


\title{
Resumen
}

En el mundo actual, las tecnologías sociales se han convertido en alternativas para la solución de problemas estructurales de la sociedad, de forma simple, de bajo costo, replicables y apoyadas por instituciones. Ellas están fundamentadas en dos bases para su propagación: la participación de personas de las comunidades que las desarrollan, y la sostenibilidad en las soluciones presentadas. En Brasil y países vecinos, se encuentran ejemplos de tecnologías sociales, certificadas por la Fundación Banco do Brasil, que generan soluciones sociocristianas. Por el perfil asociativo y solidario, esas tecnologías sociales son mecanismos que promueven la autogestión, factor relevante, en tiempos de crisis económica. El objetivo de esta investigación es analizar el desarrollo de los proyectos de tecnologías sociales, de forma que puedan ser replicados en otras comunidades de América Latina, en soluciones socio creativas. La metodología comprende la investigación bibliográfica de contextos temáticos. Las cuestiones de las comunidades de fronteras, pueden ser comprendidas por la semejanza de las dificultades territoriales, que se ralentizan por factores culturales. Se analiza el potencial de compartir las tecnologías sociales en inteligencia socio creativa. Esta investigación es un estudio de las posibilidades de generar soluciones en conjunto, en un mundo donde, las opiniones diferentes de cuestiones políticas y culturales, generan conflictos y exclusión. La inteligencia socio creativa propone la conexión de la diversidad de miradas y pensamientos, incluso opuestos, para crear realidades más inclusivas y sostenibles. Los modelos de tecnologías sociales pueden promover el diálogo entre comunidades, incluso entre fronteras, en experiencias similares. Uno de los ejemplos es el proyecto "10caminos" que propone solución en generación de ingresos para las poblaciones en situación de vulnerabilidad económica al crimen. Tiene como objetivo organizar el primer distrito creativo en la triple frontera Brasil, Argentina y Paraguay. Así, en Foz do Iguaçu / Brasil, fue creado el primer núcleo en la comunidad de Vila C - una tecnología social en modelo socio creativo. Se espera que éste se convierta en un distrito creativo, un referencial de la triple frontera latinoamericana.

Palabras clave: Tecnologías sociales, soluciones socio creativas, frontera, generación de ingresos, inclusión social.

\begin{abstract}
In today's world, social technologies have become alternatives for solving structural problems of society, in a simple, low-cost way, replicable and supported by groups of people. They have two bases for its propagation: the participation of people from the communities that develop them and the sustainability in the presented solutions. In Brazil and neighboring countries, there are examples of social technologies, certified by the Banco do Brasil Foundation, which generate socio-creative solutions. For the associative and solidarity profile, these social technologies are mechanisms that promote self-management, a relevant factor in times of economic crisis. The objective of this research is to analyze the development of social technology projects so that they can be replicated in other Latin American communities, in socio-creative solutions. The methodology is carried out by the bibliographic research of thematic contexts. The issues of border communities can be understood by the similarity of territorial difficulties, which are mitigated by cultural factors. This research deals with the potential of sharing social technologies in socio-creative intelligence. It is the possibility of generating solutions together, in a world where different opinions of political and cultural issues generate conflicts and exclusion. Sociocreative intelligence proposes the connection of the diversity of looks and thoughts, including opposites, to create more inclusive and sustainable realities. Models of social technologies can promote dialogue between communities, including across borders, in similar experiences. One of the examples is the project "10Caminhos" that proposes a solution in income generation for populations in situations of economic vulnerability, with the goal of organizing the first creative district in the triple border Brazil, Argentina and Paraguay. Thus, in Foz do Iguaçu / Brazil, the first nucleus was created in the community of Vila C - a social technology in socio-creative model. It is hoped that this will become a creative district, a benchmark of the Latin American triple frontier.
\end{abstract}

Keywords: Social technologies, socio-creative solutions, frontier, income generation, social inclusion. 


\section{Introdução}

O mundo vem sofrendo muitos impactos. As crises econômicas, sociais, ambientais, políticas, entre outras, surgem no meio urbano e desestruturam até mesmo pequenas comunidades. As pessoas se sentem desamparadas e se unem em busca de soluções. Por iniciativas de grupos sociais que atuam nos territórios afetados surgem ações contrárias aos efeitos degenerativos das desigualdades humanas. São atitudes solidárias que, por experiência de vida, conhecimento tecnológico e/ou científico, se convergem ao entorno de um propósito comum. Essas iniciativas se denominam tecnologias sociais.

Segundo Dagnino (2010) e Kliass (2012), as tecnologias sociais se popularizaram como alternativas para a resolução de problemas estruturais das camadas mais excluídas da sociedade, na medida em que apresentam soluções modernas, simples e de baixo custo. Estão fundamentadas em duas proposições importantes para sua propagação: a participação das pessoas das comunidades que as desenvolvem e a sustentabilidade nas soluções apresentadas, por meio do estímulo à formação de grupos sociais e do associativismo.

De acordo com Mourão (2017), existem exemplos de tecnologias sociais que, em termos gerais, proporcionam ganhos expressivos para a sociedade, conceituados pelas necessidades temporais, condições locais e contextos culturais. Esse fato ocorre desde a recuperação de saberes tradicionais até a apropriação de conhecimento popular, em maior grau de elaboração científica. Dessa forma, existe certa elasticidade na abrangência do conceito: desde a simples preocupação com o caráter social da utilização da tecnologia gerada até uma abordagem em que o processo de produção da tecnologia tenha em si mesmo incorporado a preocupação com a dimensão social.

A abrangência social pode se caracterizar como um processo inovador. Conforme Manzini (2008), a inovação social deve encontrar potência e determinação dentro das iniciativas locais. Sem dúvida, é um papel estratégico na busca deste novo panorama social. No Brasil e países vizinhos, constatam-se exemplos de tecnologias sociais, certificadas pela Fundação Banco do Brasil, que geram soluções sociais a partir de ações criativas desenvolvidas pela comunidade.

Pelo perfil associativo e solidário, essas tecnologias sociais são mecanismos que promovem a autogestão, fator relevante em tempos de crise econômica. O objetivo dessa pesquisa é analisar o desenvolvimento dos projetos de tecnologias sociais de forma que possam ser replicados em outras comunidades da América Latina, em soluções sociocriativas. 
A metodologia se realiza pela pesquisa bibliográfica de contextos temáticos. Para Gil (2002, p.44), pesquisa bibliográfica “[...] é desenvolvida com base em material já elaborado, constituído principalmente de livros e artigos científicos". Dessa forma, buscam-se fundamentações dos conceitos de tecnologias sociais, atividades sociocriativas e comunidades, como base para os estudos de caso citados pelo Banco de Tecnologias Sociais da Fundação Banco do Brasil.

Além do território brasileiro, as questões das comunidades de fronteira podem ser compreendidas pela semelhança das dificuldades abrandadas por fatores culturais. Aborda-se nessa pesquisa o potencial de compartilhamento das tecnologias sociais em atividades sociocriativas.

\section{Tecnologias sociais}

Busca-se inicialmente o significado da palavra 'tecnologia', como um conjunto de conhecimentos, processos e métodos empregados em diversos ramos, em definições generalizadas. A palavra tecnologia pode ser compreendida como uma atividade social, organizada e estruturada em níveis e contexto prático (BAUMGARTEN, 2006).

A adesão do termo 'social' à tecnologia disponibiliza todo esse conjunto de conhecimentos, processos e métodos, para a sociedade, com o objetivo de efetivar e expandir os direitos. O complemento social, associado à palavra tecnologia, traz a dimensão socioambiental e a construção de processos democráticos, inclusivos, visando solucionar as necessidades da população, para a esfera do desenvolvimento tecnológico (ITS, 2007).

A Comissão de Constituição de Justiça e de Cidadania aprovou, em 16 de agosto de 2017, em caráter conclusivo, o Projeto de Lei 3329/15 do Senado Federal do Congresso Nacional do Brasil, que cria a Política Nacional de Tecnologia Social (PNTS), conforme Câmara dos Deputados (2017). Na justificação da lei, destaca-se que as tecnologias sociais demonstram uma importância estratégica, devido ao seu potencial de estímulo ao desenvolvimento econômico e social. O texto ainda destaca o potencial que vem sendo desperdiçado, privando grande parcela da população brasileira de bem-estar e de melhoria na qualidade de vida e impedindo o desenvolvimento produtivo e econômico que deriva da aplicação e da disseminação de tecnologias sociais.

Conforme Maciel e Fernandes (2011) registram-se as tecnologias sociais em função política, a partir das iniciativas de Mahatma Ghandi, líder da Índia, entre os anos de 1924 e 
1927. Uma delas ocorreu pela utilização do processo de fiação manual, como forma de lutar contra as injustiças sociais prevalentes naquele país.

Em todo o mundo, surgem exemplos de tecnologias sociais, independente do termo empregado, pois o que conta é o conceito que está em construção, se adaptando as necessidades e aspectos culturais de diferentes povos. A exclusão social é um fato crescente em todo o planeta, causado pelas diferenças internas e externas em cada país, especialmente nos países mais pobres (ESTIVILL, 2003).

No Brasil, as tecnologias sociais, entre outras instituições de apoio, recebem apoio do Estado através da FBB - Fundação do Banco do Brasil, que atua no campo da Ciência e Tecnologia apoiando projetos sociais e de pesquisa. Em 2001, o programa BTS - Banco de Tecnologias Sociais foi efetivado com objetivo de investir na captação e difusão de tecnologias implementadas, reaplicáveis e efetivas, para resolução de problemas sociais. Para estimular a difusão das tecnologias sociais no território brasileiro, a FBB criou o Prêmio Nacional de Tecnologias Sociais e, através das parcerias institucionais, está investindo recursos em novas propostas.

\section{Atividades sociocriativas}

Segundo Alencar (2007), todo ser humano tem potencial criativo, sendo que a criatividade é um fenômeno complexo e multifacetado. O processo criativo é condicionado às ações de indivíduos, uma vez que grande parte da responsabilidade de criar advém das pessoas. Dessa forma, as atividades criativas podem emergir de um grupo social que se fundamentam em um modelo estrutural sistêmico, compartilhado e colaborativo.

As redes sociocriativas, mais do que sociotécnicas, se estabelecem em contribuições mútuas. "E é isto que dá sustentação aos esforços de captação e interpretação dessas idéiasforça, bem como de outras fontes de inspiração, informação e conhecimento" (PIRES, ALBAGLI, 2012, p.113).

Para Gardner (1995), a inteligência é algo mais complexo, elas são múltiplas e estão para além de conjunto de capacidades relativamente independentes, apresentando áreas que dinamizam a capacidade de raciocínio.

A inteligência sociocriativa é uma linguagem de padrões e princípios para pensar e praticar em empreendimentos individuais e coletivos, em geração de projetos e sistemas de organizações. Trata-se de uma metodologia desenvolvida pelo Laboratório de inteligência 
sociocriativa. Em contexto interdisciplinar, se aplica em estrutura de quadro etapas de atividades, que são: sentido, propósito, método, aprendizado, conforme Martinez (2015).

Consideram-se também as abordagens socioculturais, em que a cultura, as relações sociais contribuem para o aprendizado humano, tanto quanto o ser humano desenvolve as relações e a cultura, conforme Ministério da Cultura (2017):

\footnotetext{
Nossa cultura, nossos pensamentos, práticas e aprendizados constroem as relações sociais ao mesmo tempo em que são construídos por elas. Isso naturalmente envolve um processo de aprendizagem individual e coletiva que vai tecendo as relações sociais e também as relações do homem com o meio ambiente econômico, cultural, social, político e natural (MINC, 2017, p. 6).
}

Dessa forma, o contexto das relações sociais pode definir inclusive as características do meio onde o grupo social está inserido. Peruzzo e Volpato (2009) dizem que o termo comunidade refere-se a presença e participação pelos laços familiares, locais e contextuais, nos quais o sentimento de pertencimento aparece como elemento fundamental na sua formação.

Percebe-se que a motivação das pessoas gera organizações e grupos que atuam na mesma atividade e compartilham os resultados positivos e negativos dos negócios, são denominados grupos solidários de autogestão. Estes empreendimentos, conforme Costa (2008) são sustentados pela força no potencial no trabalho coletivo que os compõem.

\section{Atuações em comunidades de fronteira: Brasil/Uruguai/Argentina}

Um dos exemplos é o projeto "10caminhos" que propõe solução em geração de renda para as populações em situação de vulnerabilidade econômica, com o objetivo de organizar o primeiro distrito criativo na tríplice fronteira Brasil, Argentina e Paraguai, especificamente em Foz do Iguaçu. Por abrigar as Cataratas do Iguaçu, a cidade é um grande pólo turístico. As Cataratas são um patrimônio natural brasileiro e argentino, podendo ser visitadas a partir dos dois países, conforme site da Unila.

Contudo, com entrada para o Brasil, há uma grande potencia de fluxo comercial na Ciudad del Este (Cidade do Leste), divisa com o Paraguai. Diariamente milhares de carros, motos, ônibus, vans e pedestres cruzam a Ponte Internacional da Amizade para trabalhar, fazer compras ou passear. Segundo dados divulgados pelo Centro Universitário UDC, aproximadamente 40.000 veículos atravessam a ponte por dia, entre idas e vindas - 
Brasil/Paraguai. Em 2017, 455.628 pessoas atravessaram a ponte da amizade, de acordo com Buche (2017). Na Figura 1, observa-se o fluxo de veículos constante na Ponte Internacional da Amizade, fronteira entre o Brasil e Paraguai.

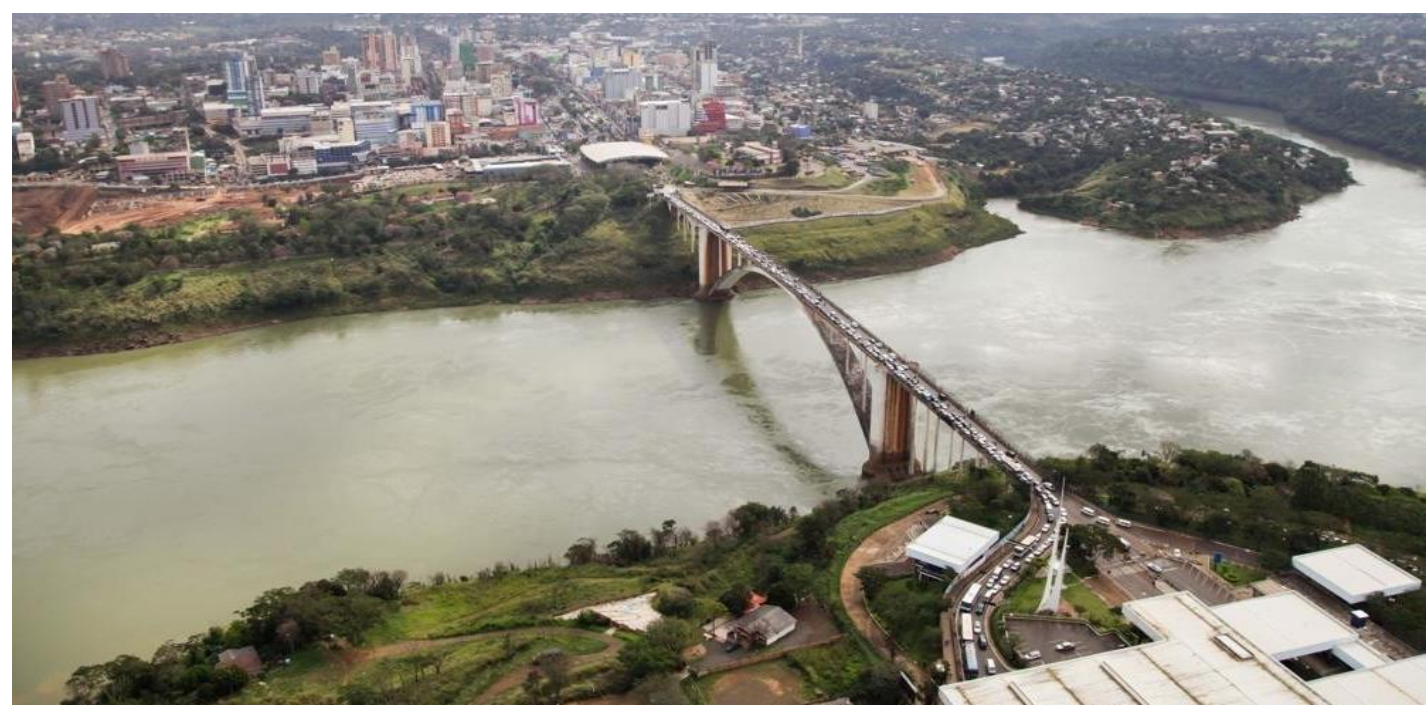

Figura 1 - Ponte Internacional da Amizade - Brasil/Paraguai.

Fonte: Kiko Sierich/Gazeta do Povo/ Arquivo, 2018.

Ao mesmo tempo em que há tanto fluxo comercial, há também populações carentes. A região necessita de investimentos sociais e urbanos, condizentes à diversidade cultural no entorno. Assim, em Foz do Iguaçu/Brasil, foi criado o primeiro núcleo na comunidade da Vila C - uma tecnologia social em modelo sociocriativo. Conforme informações do Projeto 10Caminhos, pretende-se ainda criar mais nove núcleos de trabalho, além do Coletivo de Costura, que serão os "10caminhos" para geração de renda com a troca de saberes da comunidade.

De acordo com Banco de Tecnologias Sociais da Fundação Banco do Brasil, a proposta apresenta um modelo de atuação do design participativo, que confere aos processos criativos e produtivos dos participantes, a possibilidade destes buscarem de forma criativa, um sistema de produção de maneira horizontal e participativa.

É também um processo de construção coletiva de projetos de produtos e processos produtivos. O coletivo é formado por uma equipe de oito senhoras da comunidade que estão articuladas em regime de produção participativa e com partilha solidária das responsabilidades do negócio solidário, com a colaboração de professores e estudantes da Unila. A ação é desenvolvida no prédio do Conselho Comunitário, onde há 19 máquinas de costura em operação e um espaço para a criação e produção (UNILA, 2017). 
Utilizando as ferramentas do design social, o projeto busca, atualmente, criar uma coleção de adornos com o aproveitamento de materiais apreendidos pelo controle fiscal da Receita Federal, doações de materiais dos projetos Nandeva e do Parque Tecnológico Itaipu.

Na Figura 2, apresentam-se as pioneiras do projeto, algumas e o ambiente de trabalho de produção.

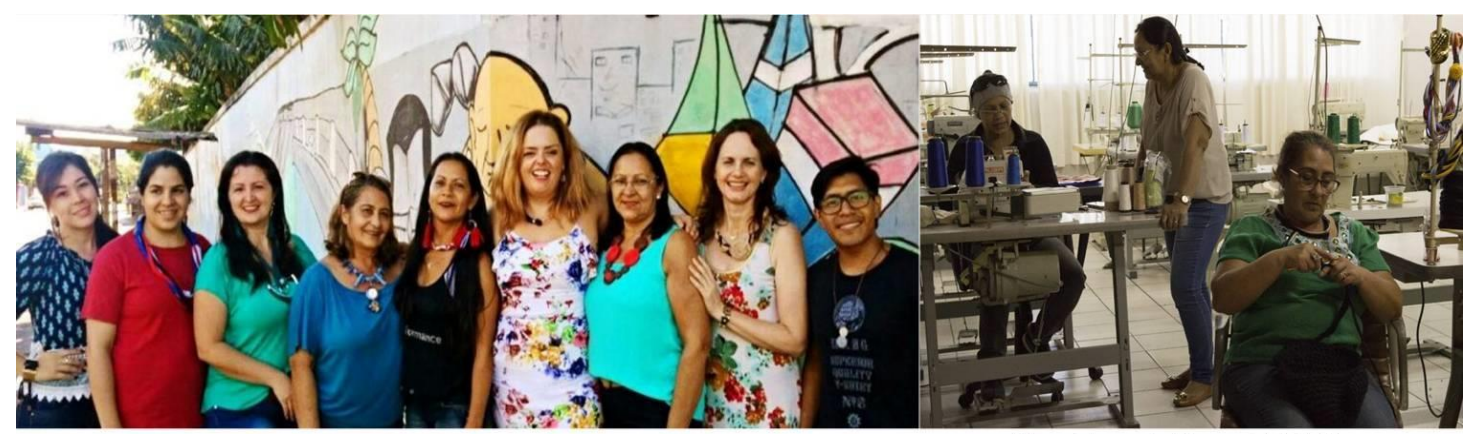

Figura 2 - Equipe de trabalho do Projeto "10Caminhos" e ambiente de trabalho.

Fonte: 10Caminhos, 2018.

As participantes trocam conhecimento em atividades artesanais, transformando pedaços de materiais apreendidos e recicláveis em peças exclusivas, considerando inclusive, as influências do entorno urbano e natural da cidade.

Na figura 3 observa-se um dos adornos feminino, desenvolvido pelas habilidades artesanais, com a participação do design, a partir de partes de um objeto industrializado.

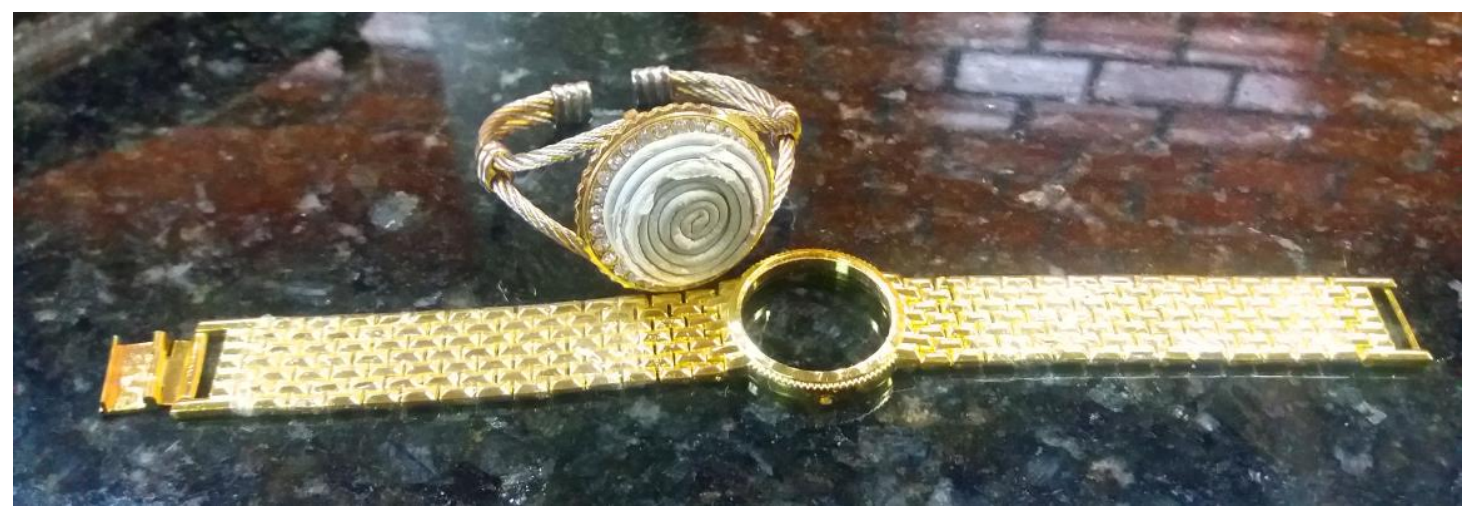

Figura 3 - Bracelete do catálogo de produtos do Projeto 10Caminhos.

Fonte: 10Caminhos, 2018.

$\mathrm{Na}$ figura 3, apresentam-se as peças do catálogo, peças exclusivas de materiais eletrônicos e retalhos de tecidos e couros. 


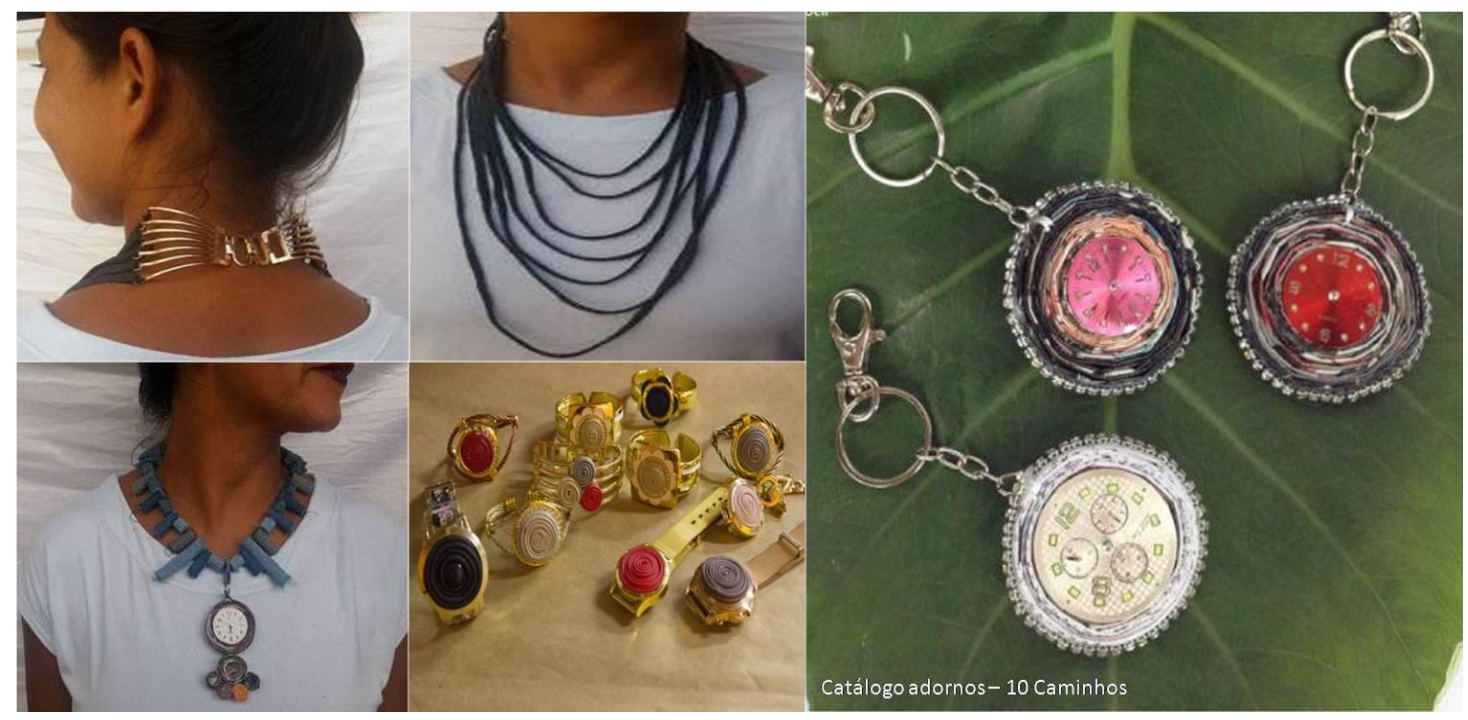

Figura 3 - Peças do catálogo de produtos do Projeto 10Caminhos.

Fonte: 10Caminhos, 2018.

Além disso, as peças de adornos estão expostas no catálogo virtual do projeto, que busca recursos em plataforma colaborativa para sua expansão. Contudo, tratando-se de aproveitamento de materiais apreendidos e controlados pela Receita Federal, os produtos serão sempre exclusivos.

\section{Analise dos Resultados e considerações}

O projeto se organiza em economia solidária, conferindo a possibilidade de criação de relações produtivas pela e na coletividade, relacionando a produção coletiva com o "bem viver", utilizando o Índice de Felicidade Bruta, como indicador qualitativo dos resultados.

Modelos de tecnologias sociais podem promover o diálogo entre comunidades, inclusive entre fronteiras, em experiências semelhantes. A proposta desse projeto é adaptável a outras comunidades das três fronteiras Brasil, Argentina e Paraguai. Acredita-se que o diálogo entre as comunidades poderá gerar um novo modelo de desenvolvimento para a região, aproveitando os recursos e os saberes locais.

Com base neste novo cenário, que possibilita a readequação dos métodos, fundamentase o potencial de criatividade e diversidade do design brasileiro. As possibilidades de expansão desassociadas do mercado buscam afastar velhas e conhecidas práticas embasadas em meras suposições. A aceitação do gosto popular e dos produtos artesanais, com melhoria no processo de produção e acabamento dos artefatos e adornos, facilita a inserção dos produtos artesanais no mercado. 
Existem programas de incentivo ao desenvolvimento de novas propostas, mas como em outras áreas e pela cultura de fomentos nacional, sem investimentos e recursos para longo prazo, as articulações com as empresas só atendem a interesses pontuais, como projetos destinados a grupos e associações solidárias. Contudo, pela diversidade do território brasileiro e mesmo com as dificuldades vivenciadas pela sociedade, a criatividade reflete um retrato multifacetado, com soluções expressivas do design.

Espera-se que a expansão desse projeto possibilite a melhoria da qualidade de vida na comunidade, atuando contra a marginalidade e o desemprego, tornando-a distrito criativo, um referencial da tríplice fronteira Latina Americana.

\section{Referências}

ALENCAR, E. M. L. S. Criatividade no Contexto Educacional: Três Décadas de Pesquisa. Revista Psicologia: Teoria e Pesquisa, Vol. 23 n. especial, p. 045-049, 2007.

BAUMGARTEN, M. Tecnologia. In: CATTANI, A.; HOLZMANN, L. Dicionário de trabalho e tecnologia. Porto Alegre: Ed. UFRGS, p. 288-292, 2006.

BUCHE, Patrícia. Ponte da Amizade: a fronteira mais movimentada do país. Postado em: 07 Dez, 2017. Disponível em: <https://www.100fronteiras.com/ponte-da-amizade-fronteiramais-movimentada-do-pais/>. Acesso em 28 dez. 2018.

CAMARA DOS DEPUTADOS. Ciência e Tecnologia. Câmara aprova criação da Política Nacional de Tecnologia Social. Postado em: 16 ago. 2017. Disponível em: $<$ http://www2.camara.leg.br/camaranoticias/noticias/CIENCIA-ETECNOLOGIA/539215-CAMARA-APROVA-CRIACAO-DA-POLITICA-NACIONALDE-TECNOLOGIA-SOCIAL.html>. Acesso em: 22 dez. 2018.

CARNEIRO FILHO, C. P. Tríplice fronteira Brasil-Argentina-Paraguai: transfronteirização através do crime. Revista Relações Internacionais no Mundo Atual, Curitiba, vol.2, ${ }^{\circ}{ }^{16}$, 2012.

DAGNINO, R. [Org.]. Tecnologia Social: ferramenta para construir outra sociedade. Campinas: Komedi, 2010.

DIRETORIA EXECUTIVA NACIONAL (DEN). Projeto Fronteira em Foco, Brasília: Sindicato Nacional dos Auditores-Fiscais da Receita Federal do Brasil, 2011. Disponível em:

<https://www.sindifisconacional.org.br/images/atividades/Fronteira_em_Foco/Fronteira_em _Foco.pdf>. Acesso em: 22 dez. 2018.

ESTIVILL, J. Panorama da Luta contra a Exclusão Social. Conceitos e Estratégias. Genebra: Bureau Internacional do Trabalho, Programa Estratégias e Técnicas contra a Exclusão Social e a Pobreza, 2003. 
FUNDAÇÃO BANCO DO BRASIFBB. Banco de tecnologias sociais. Disponível em: 〈www.tecnologiasocial.org.br/bts/>. Acesso em: 02 dez. 2018.

GARDNER, H. Inteligências Múltiplas: a Teoria na Prática. Porto Alegre: Artes Médicas, 1995.

GIL, A. C. Métodos e técnicas de pesquisa social. São Paulo: Atlas, 2002.

INSTITUTO DE TECNOLOGIA SOCIAL - ITS. Declaração das ONGs: ciência e tecnologia com inclusão social, 2005.

KLIASS, P. A importância da tecnologia social. In: carta maior.com.br., 2012.

MACIEL, A. L. S.; FERNANDES, R. M. C. Tecnologias sociais: interface com as políticas públicas e o Serviço Social. Revista Serviço Social \& Sociedade, São Paulo, nº 105, p. 146165, jan./mar, 2011.

MANZINI, E. Design para a inovação social e sustentabilidade: comunidades criativas, organizações colaborativas e novas redes projetuais. Rio de Janeiro: E-papers, 2008.

MARTINEZ, A. Cultura e Mercado. 10 atitudes sociocriativas. Postado em: 5 mar. 2015. Disponível em: $\quad$ http://www.culturaemercado.com.br/site/destaque/10-atitudessociocriativas/>. Acesso em: $22 \mathrm{dez} .2018$.

MINISTÉRIO DA CULTURA. Guia do Empreendedor Sociocultural. Disponível em: <https://sociocriativo.files.wordpress.com/2017/12/guia_empreendedor_sociocultural.pdf>.

Acesso em: 22 dez. 2018.

MOURÃO, N. M. Tecnologias sociais e empreendimentos criativos na Região Metropolitana de Belo Horizonte/MG. Revista Brasileira do Desenvolvimento Regional, PPGDR/Universidade Regional de Blumenau, ISSN 2317-5443, 2017. DOI: <http://dx.doi.org/10.7867/2317-5443.2017v5n1p053-067>. Acesso em: 22 dez. 2018.

PERUZZO, C. M. K. ; VOLPATO, M. O. Conceitos de comunidade, local e região: interrelações e diferença. Revista Líbero, São Paulo, v. 12, n. 24, p. 139-152, dez. de 2009. Disponível

em:

<http://www.unipublicabrasil.com.br/uploads/materiais/98faa318d1ce26fd10a1a6bc901e3e4 a26072016161928.pdf $>$. Acesso em: 22 dez. 2018.

PIRES, V. S.; ALBAGLI, S. Estratégias empresariais, dinâmicas informacionais e identidade de marca na economia criativa. Revista Perspectivas em Ciência da Informação, v.17, n.2, p.109-122, abr./jun. 2012. Disponível em: <http://www.scielo.br/pdf/pci/v17n2/a08v17n2.pdf>. Acesso em: 22 dez. 2018.

UNILA - Universidade Federal da Integração Latino-Americana. 10Caminhos. Disponível em: 〈https://www.unila.edu.br/noticias/10caminhos>. Acesso em: 22 dez. 2018. 